\title{
Utilizing the Generalized Intelligent Framework for Tutoring to Encourage Self-Regulated Learning
}

\author{
Anne M. Sinatra \\ U.S. Army Research Laboratory \\ anne.m.sinatra.ctr@us.army.mil
}

\begin{abstract}
Self-regulated learning is of particular importance to computer-based and online instruction, as students need to manage their own time and their interactions with the system. Elements of self-regulatory learning traditionally include the metacognitive strategies of the students (e.g., their knowledge of their planning, and assessment of their own progress), their management of educational goals (e.g., what information is most important to them, and should receive their primary attention), and the strategies that students use in order to study and retain the provided information $[1,2]$. By incorporating feedback and guidance within computer-based learning activities it can encourage students to engage in successful self-regulated learning with a better awareness of their own cognition, and strategies. Intelligent tutoring systems can utilize adaptive scaffolding and guidance in order to support self-regulated student learning [3]. The Generalized Intelligent Framework for Tutoring (GIFT) [4] is an opensource adaptive tutoring system framework. The included tools within GIFT can be used to structure courses which guide individuals through the learning environment and are consistent with self-regulated best practices. The current paper includes a brief review of research into self-regulated learning in the context of computer-based and adaptive instruction. Further, the authoring capabilities of GIFT are discussed, and recommendations are given for future feature additions to GIFT which will benefit instructors who wish to develop courses that facilitate self-regulated learning.
\end{abstract}

Keywords: Strategy assessment and individual differences, Self-Regulated Learning, Adaptive Tutoring, Intelligent Tutoring Systems.

\section{Self-Regulated Learning in Computerized Environments}

Self-Regulated learning involves an individual being in control of his or her own learning, and successfully retaining the material. Students who are self-regulated learners are able to employ metacognitive strategies to monitor their own learning and progress, as well as adjust their strategies accordingly to improve their outcomes. These learners are also able to both set and manage their own educational goals [2]. While the study of self-regulatory learning processes began in the classroom environment, they also have great importance in computer based and adaptive learning environments $[5,6]$. Classroom based learning is generally regulated by an instructor 
who provides structure to the learner, provides feedback, and sets a pace for the material. However, in most computer-based learning environments (CBLEs), the responsibility for the pacing of the tasks, time spent with the system, and monitoring of one's own understanding rests with the learner him or herself. In these situations selfregulated learning and the encouragement of it through techniques such as scaffolding can be an important factor into the success the individual has learning from the system [7, 8]. Some students have a natural inclination towards being self-regulated learners. However, by teaching students self-regulatory strategies and encouraging them to use them, it could have multiple benefits to the learner. First, they will learn more from the provided material, and secondly, they may retain the strategies and use them in the future. Scaffolding is an approach that has shown success in teaching and encouraging self-regulatory learning strategies in CBLEs [3].

\section{Using Scaffolding to Teach Self-Regulatory Strategies}

Similarly to self-regulation research, scaffolding research initially began in the classroom [9]. Scaffolding traditionally involves a more advanced individual (such as an adult, a teacher, or a more advanced student) providing feedback and information to a learner which can help guide them through a process or task that they would not be able to do on their own. The more advanced individual offers support to the learner, so that he or she can use their effort to concentrate on the vital elements of the task which they are capable of handling. In addition, this more advanced individual continuously monitors the state of the learner (on-going diagnosis) and makes adjustments to their own instructional strategies accordingly (calibrated support). Another key component of scaffolding is fading, which results in the gradual removal of the feedback and support provided by the advanced individual, until the student is learning on his or her own. Over time, specifically with the integration of it into computerized environments, the definition of scaffolding has shifted, and new considerations need to be made to the impact of the new environment [10]. In a computerized environment, the system and scaffolding of material takes the role of the advanced individual, or teacher, and assists in structuring learning for the individual student.

Research has examined the benefits of self-regulatory and metacognitive strategies in hypermedia, online courses, online inquiry, as well as adaptive tutoring $[7,11,12$, 13]. Interestingly, despite the greatly varying characteristics of these CBLEs, they have generally been treated similarly in research. While the term technical scaffolding [14] has been coined to describe scaffolding which involves computers and technology, future research is required to start parsing out the different effects that the varying interactivity of these environments provide. One of the reasons for this lack of research is the difficulty in creating true scaffolding (with on-going analysis, calibrated support, and fading) in computerized environments. However, tools and domain independent frameworks such as the U.S. Army Research Laboratory's Generalized Intelligent Framework for Tutoring (GIFT) provide flexibility that is conducive to engaging in self-regulatory and scaffolding research in adaptive systems $[4,15]$. 


\section{The Generalized Intelligent Framework for Tutoring (GIFT)}

GIFT is a primarily open-source, domain independent intelligent tutoring system framework, which is freely available from www.gifttutoring.org. GIFT allows individuals to use their own content to create adaptive courses and intelligent tutoring systems (ITSs). It is important to conduct research into what techniques are most effective in ITSs, to ensure that students are getting the most out of their time interacting with the system. However, it is often impractical to do investigations within ITSs due to their long development time, and lack of flexibility. Due to its domain independent and modular design, GIFT provides the ability for researchers to easily design and run experiments that traditionally would be time or cost prohibitive in ITS systems.

GIFT includes components of traditional ITS systems in the form of multiple independent modules, and a user interface [16]. The fundamental components of GIFT consist of sensor, pedagogical, learner, gateway, and domain modules. The learner's current cognitive, affective, and motivational state is represented in the learner module, and can be determined through input from the sensors, performance output from the tasks that the learner engages in the domain, as well as from surveys that the learner completes. Currently, work is being done on the pedagogical module, such that it will be able to choose appropriate instructional strategies and material for the learner based on his or her individual characteristics. In the current design of GIFT, the author of the course determines which instructional strategies are to be implemented based on the transition of learner states, which is authored into a Domain Knowledge File (DKF). The gateway module provides a link between an external training application (such as VBS2, PowerPoint, or TC3 Sim/Vmedic) and GIFT. Finally, the domain module stores all information that is specific to the current domain and course that is being used in GIFT. This domain content can be provided by the individual author of the GIFT course.

GIFT 4.0 includes a suite of authoring tools which provide the ability to author courses (the Course Authoring Tool, or CAT), author adaptive feedback and instructional strategies (the Domain Knowledge File Authoring Tool, or DAT), and author surveys (the Survey Authoring System, or SAS). Additionally, the researcher/instructor can include sensors (using the Sensor Configuration Tool, or SCAT), and adaptive branching course flows based on an individual student's characteristics (using the Metadata Authoring Tool, or MAT and Pedagogy Configuration Authoring Tool, or PCAT). Currently, the authoring tools which are included with GIFT all follow the same structure and are based on .xml editors.

In order to create a new course, the instructor/author uses the CAT to design their desired course flow. Nodes can be added and expanded within the CAT to add transitions, surveys, external training applications, and guidance that is provided to the learners. The instructor/author can also link training applications in the course to specific DKF files that they have created. After completing the creation of a course, it can be exported using the Export Tool, which allows it to run on a computer without needing to install the full version of GIFT. 
The included tools are usable, however, they take time to learn, and may initially appear intimidating to non-computer scientists. See Figure 1 for a screenshot example of the CAT. Future versions of GIFT will include a more user-friendly version of the CAT, which will include a wizard and drag/drop functionality. Moving forward, one of the goals of GIFT is to become easier to use, and encourage non-computer scientists to author material within GIFT.

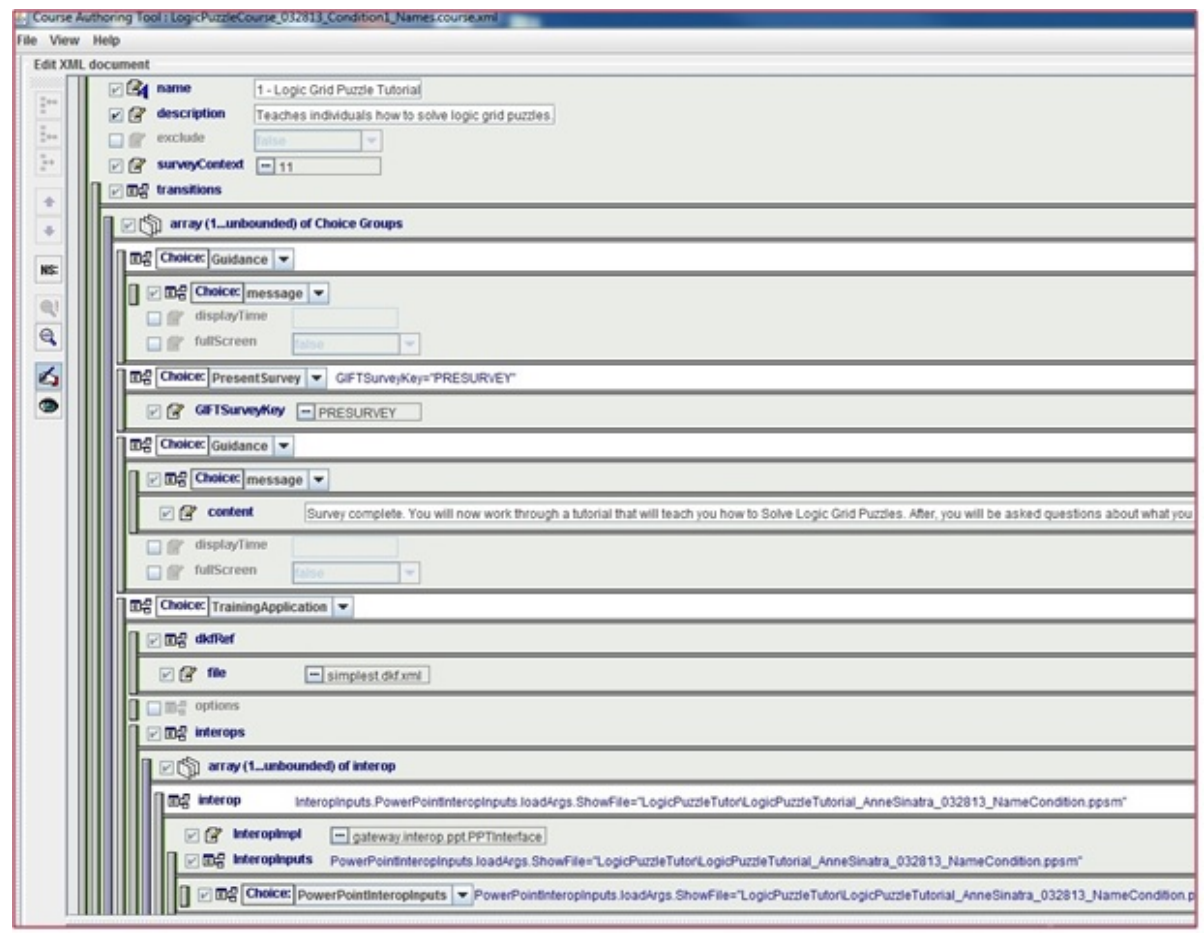

Fig. 1. An example of a course loaded in the Course Authoring Tool (CAT)

\section{Utilizing the Current Features of GIFT to Encourage Self-Regulated Learning}

GIFT provides the ability to author a complete ITS, which can assess the current performance level of individuals and adjust feedback and instruction based on it. These specific areas of interest are defined by the instructor/author as concepts in a DKF. Currently, these files can be generated for use with the supported external training applications (VBS2, PowerPoint, and TC3 Sim/Vmedic) through using the DAT. Additionally, the SIMILE Workbench Tool allows for development of DKFs for use with TC3 Sim/Vmedic, and current work is being done to expand its compatibility to additional training applications. In GIFT the states of the user can be defined as Below Expectation, At Expectation, and Above Expectation. These states are adjusted 
based on the performance metrics that have been authored into the DKF, and can include data linked to surveys and tasks within a game environment.

Scaffolding can be achieved through monitoring the user's current state (Below Expectation, At Expectation, or Above Expectation). If the performance of the individual transitions from Above Expectation to At Expectation, or At Expectation to Below Expectation, responses and feedback can be given to the student. These responses can be given in the form of written text on the screen, or spoken audio files over the computer's speakers. Additionally, GIFT is integrated with the Media Semantics Character, which allows feedback to be provided from an avatar if the author wishes for it to be. By including an avatar it may make an additional connection with the student, and they may be able to identify with it as an instructor, or co-learner. The inclusion of this social presence may assist in encouraging the student to engage with the program, and regulate their own learning.

This monitoring of the user's state, and adjustment for it are consistent with the components of scaffolding in which the teacher monitors and assesses the current state of the learner (on-going diagnosis), and adjusts specifically for their performance (calibrated support). The feedback that is provided to the learner after a transition in state is authored in the DKF, and can be used to promote self-regulatory learning, as well as best practices. Through this feedback, the GIFT course designer can provide suggestions on how to progress, ask the individual to think about why they have made their decisions, or ask them to assess their current progress. By prompting individuals to engage in these tasks it can structure their learning, as well as require them to elaborate and review what they have learned. By consistently offering this feedback, it can encourage the learners to engage in the tasks even when they encounter different systems and materials in the future. Multiple choice questions can be provided to the student between instruction in order to get them to think about and retrieve information that they learned. These assessments can be automatically graded. If used in conjunction with a training application, these grades can be used to determine the state that the individual is in. The state can then be used to identify concepts that need to be reviewed, or that need the learner needs to receive additional tutoring on.

Additionally, the instructor has the ability to export the states that the learner was in during the tutoring session. This information can then be used after the fact to examine if the interventions were successful or helpful to the individual student. The more information that can be gathered about the learner's state, the more helpful the feedback can be that is provided to them.

\section{Recommendations}

There are improvements that can be made to GIFT which can expand its current capabilities, and offer more options to authors/instructors interested in encouraging selfregulated learning. Currently, adaptive rules are authored in a DKF, which is then associated directly with a training application. Therefore, adaptive feedback and paths can only be offered within the training application. In the near future GIFT will have 
an ability to provide specific content based on the individual learner's characteristics. However, it would be beneficial to have the ability to immediately score a survey and use that score to determine the feedback that is offered to the student, or the path of instruction that GIFT will provide. This would result in more tailored material, and strategies that can be used to scaffold the individual student.

Elements of AutoTutor [17] have been integrated within GIFT, and one of the functions is the ability to author dialogues that are presented to the learner. The learner is prompted to answer a question, and then the response is examined. If the learner response does not meet the authored criteria, hints can be provided. This method can encourage metacognition, and self-regulation. However, currently, the learner is able to interact with the tutor and provide feedback, but the interaction and data is not available for export. It would be beneficial for the instructor to be able to see how many attempts it took the individual student to answer a specific question properly, and to be able to examine the specific text that he or she entered into the program. This could help the instructor examine misconceptions, and have a better understanding of the student's thought process. See Figure 2 for an example of an AutoTutor dialogue in GIFT.

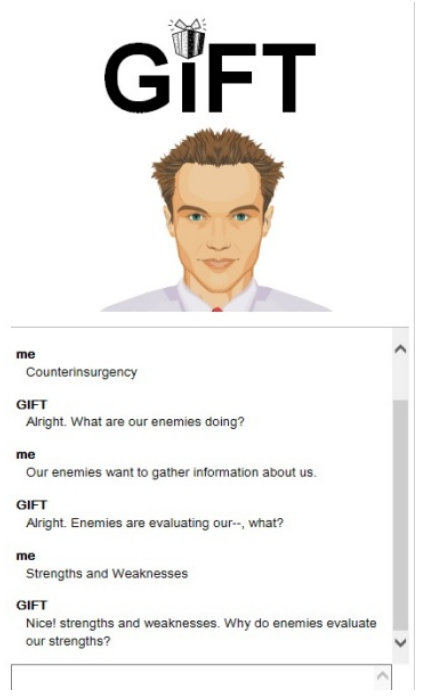

Fig. 2. Example screenshot of a dialogue included in the GIFT Course titled "COIN AutoTutor Session Example". The information from the student's interaction is not currently available to the instructor after course completion.

An additional recommendation, which is already being worked on, is making GIFT more user-friendly. The instructor/author currently has the ability to implement a number of different strategies in GIFT. However, there can sometimes be a learning curve to using the authoring tools in GIFT. Adaptive feedback, in the form of a DKF, is one of the more difficult pieces to author, and the DAT would benefit from a more straightforward or simplified interface. The expansion of the external SIMILE 
Workbench Tool to multiple training applications should assist in reducing the workload of the instructor/author who is creating the DKF files. Moving toward an integrated Course Authoring Tool which provides ties to the other necessary tools and offers a help setting would also be extremely beneficial. Many instructors/authors may not feel comfortable using the current .xml editor tools, but the ability to quickly understand and use a new more intuitive authoring tool will be of great benefit to noncomputer scientists.

\section{Conclusions}

Self-regulated learning is very important in computer based and adaptive systems. Without the presence of an instructor to maintain the pacing and flow of a course, it is up to the individual to monitor/progress their own learning, and make sense of the material. According to the Cognitive Transformation Theory, the process of the learner making sense of the provided material and building/adjusting their mental models of how the concepts work is vital to learning outcomes [18]. This is particularly important in a CBLE where an instructor is not present to help guide the learning process. Using questions, metacognitive prompts and feedback during the computerized learning process can encourage the individual to engage in self-regulatory processes that will assist them in their current learning, as well as adjustment of their mental models and may provide benefits for their future interaction with CBLEs and studying. As a domain independent intelligent tutoring framework, GIFT provides the ability for an instructor/author to create a course that is adaptive to the individual learner, and to offer self-regulatory feedback and guidance throughout the learning experience. As GIFT continues to develop it will continue to be a useful tool for encouraging and teaching self-regulatory best practices and skills.

Acknowledgements. The author would like to thank Dr. Benjamin Goldberg and Dr. Robert Sottilare for providing comments on the paper. Research was sponsored by the Army Research Laboratory and was accomplished under Cooperative Agreement Number W911NF-12-2-0019. The views and conclusions contained in this document are those of the author and should not be interpreted as representing the official policies, either expressed or implied, of the Army Research Laboratory or the U.S. Government. The U.S. Government is authorized to reproduce and distribute reprints for Government purposes notwithstanding any copy-right notation herein.

\section{References}

1. Pintrich, P.R., DeGroot, E.V.: Motivational and self-regulated learning components of classroom academic performance. Journal of Educational Psychology 82(1), 33-40 (1990)

2. Zimmerman, B.J.: Self-regulated learning and academic achievement: An overview. Educational Psychologist 25(1), 3-17 (1990)

3. Lajoie, S.P.: Extending the scaffolding metaphor. Instructional Science 33, 541-557 (2005) 
4. Sottilare, R.A., Brawner, K.W., Goldberg, B.S., Holden, H.K.: The Generalized Intelligent Framework for Tutoring (GIFT). Army Research Laboratory (2012)

5. Azevedo, R., Hadwin, A.F.: Scaffolding self-regulated learning and metacognition - Implications for the design of computer-based scaffolds. Instructional Science 33, 367-379 (2005)

6. Winters, F.I., Greene, J.A., Costich, C.M.: Self-regulation of learning within computerbased learning environments: A critical analysis. Educational Psychology Review 20, 429444 (2008)

7. Azevedo, R., Cromley, J.G., Seibert, D.: Does adaptive scaffolding facilitate students' ability to regulate their learning with hypermedia? Contemporary Educational Psychology 29, 344-370 (2004)

8. Dabbagh, N.: Scaffolding: An important teacher competency in online learning. Tech.Trends 47(2), 39-44 (2003)

9. Wood, D., Bruner, J., Ross, G.: The role of tutoring in problem solving. Journal of Child Psychology \& Psychiatry \& Allied Disciplines 17(2), 89-102 (1976)

10. Puntambekar, S., Hubscher, R.: Tools for scaffolding students in a complex learning environment: What have we gained and what have we missed? Educational Psychologist 40(1), $1-12(2005)$

11. El Saadawi, G., Azevedo, R., Castine, M., Payne, V., Medvedeva, O., Tseytlin, E., et al.: Factors affecting feeling-of-knowing in a medical intelligent tutoring system: the role of immediate feedback as a metacognitive scaffold. Advances in Health Science Education $15,9-30(2010)$

12. Holden, H.K., Sinatra, A.M.: A guide to scaffolding and guided instruction. In: Sottilare, R., Graesser, A., Hu, X., Goldberg, B. (eds.) Design Recommendations for Intelligent Tutoring Systems. Instructional Strategies, vol. 2 (in press, 2014)

13. Zhang, M., Quintana, C.: Scaffolding strategies for supporting middle school students' online inquiry processes. Computers \& Education 58, 181-196 (2012)

14. Yelland, N., Masters, J.: Rethinking scaffolding in the information age. Computers \& Education 48, 362-382 (2007)

15. Sottilare, R.A., Holden, H.K.: Motivations for a Generalized Intelligent Framework for Tutoring (GIFT) for authoring, instruction and analysis. In: AIED 2013 Workshops Proceedings, vol. 7, pp. 1-9 (2013)

16. Sottilare, R.A., Graesser, A.C., Hu, X., Holden, H.: Preface. In: Design Recommendations for Intelligent Tutoring Systems, vol. 1, pp. ii-xiii (2013)

17. Graesser, A.C., Wiemer-Hastings, K., Wiemer-Hastings, P., Kreuz, R.: AutoTutor: A simulation of a human tutor. Cognitive Systems Research 1(1), 35-51 (1999)

18. Klein, G., Baxter, H.C.: Cognitive transformation theory: Contrasting cognitive and behavioral learning. In: The Interservice/Industry Training, Simulation \& Education Conference (I/ITSEC), vol. 2006(1). National Training Systems Association (2006) 